\title{
Body composition and energy expenditure in patients with chronic obstructive pulmonary disease
}

\section{Frode Slinde}

\section{Department of Clinical Nutrition, Sahlgrenska Academy, Göteborg University, Göteborg, Sweden}

The prevalence of chronic obstructive pulmonary disease (COPD) is increasing in Sweden as well as worldwide. The main cause of the disease is cigarette smoking. Almost $50 \%$ of all patients with COPD become underweight. The questions addressed in the thesis were: (i) Does body composition measured by bioelectrical impedance predict mortality in patients with COPD? (ii) How large a variation can be seen in body composition measured by bioelectrical impedance during $24 \mathrm{~h}$ ? (iii) How much energy do underweight patients with COPD expend when they are living their normal lives at home and during a physiotherapy programme?

Methods used were bioelectrical impedance analysis and dual-energy X-ray absorptiometry to assess body composition, doubly labelled water to measure total daily energy expenditure, indirect calorimetry to measure basal metabolic rate (BMR), and 7 day dietary registrations to measure energy intake.

In a sample of patients with COPD, who had been included in a 1 year multidisciplinary rehabi-

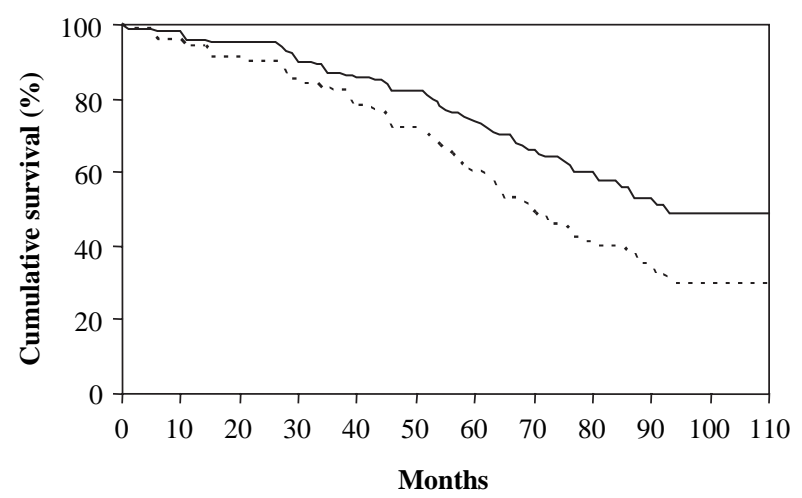

Fig. 1. Cumulative survival in a group of patients with chronic obstructive pulmonary disease categorized by fat-free mass index (FFMI) $(n=86)$. FFMI $\left(\mathrm{kg} / \mathrm{m}^{2}\right) ;->16.6$ (median); $\cdots \cdots<16.6$ (median). litation programme, those patients with a high proportion of fat-free mass (measured by bioelectrical impedance) lived longer than those with a low proportion of fat-free mass (1). As shown in Fig. 1, $40 \%$ of the patients with a low fat-free mass index (FFMI) had died after 5 years (60 months) compared with $22 \%$ of the patients with FFMI higher than the median of the total group. Other factors found to be independent predictors of mortality in the patient group were age and gender.

It is important to standardize the measurements of body composition by bioelectrical impedance ( 2 , 3). Measurements should be taken in the fasting state after the subject has been in the supine position for $10 \mathrm{~min}$. Lack of standardization had large effects on the calculated body fat percentage in a group of healthy volunteers. FFMI was shown to have smaller variation during the day, mainly because of the use of body height, which is a more stable variable than body weight, which is used to calculate body fat percentage.

Underweight patients with COPD were found to have a large variation in energy expenditure (4), both BMR and energy expenditure for physical activity. In 10 patients with severe COPD and BMI $<21 \mathrm{~kg} \mathrm{~m}^{-2}$, BMR varied from 88 to $135 \mathrm{~kJ} \mathrm{~kg}^{-1}$ body weight. Existing prediction equations of BMR were shown to predict BMR with poor precision in these patients. A variation in total daily energy expenditure from 1.2 to 1.8 times BMR was reported.

As illustrated in Fig. 2, some patients increased their total daily energy expenditure during 2 weeks of training with a physiotherapist, while others decreased their total daily energy expenditure (5). Energy intake of the patients measured by a 7 day dietary record did not correspond to their energy 


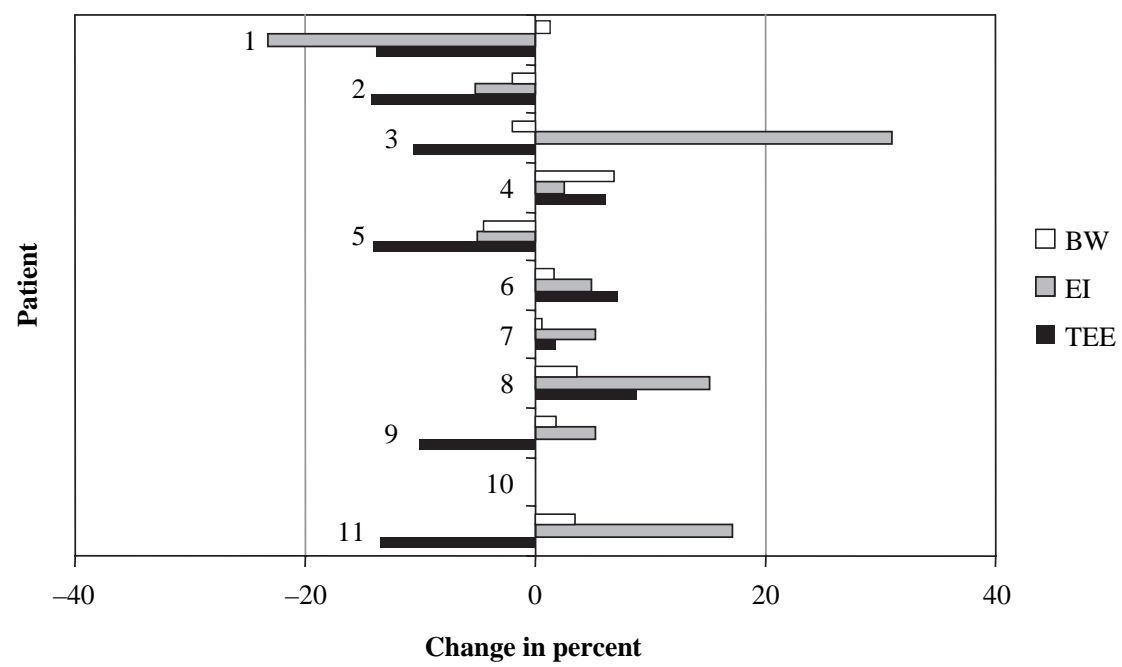

Fig. 2. Change (\%) in body weight (BW), energy intake (EI) and total daily energy expenditure (TEE) from control to physiotherapy period in 10 underweight patients with chronic obstructive pulmonary disease. (Patient number 10 did not complete the physiotherapy period).

expenditure, since in most cases these two measurements do not agree.

In conclusion, bioelectrical impedance may be a prognostic tool in COPD, but the measurements need to be standardized. Patients with COPD at the same level of disease and body weight may have totally different levels of energy expenditure. The energy requirement of underweight COPD patients should therefore be assessed individually. New methods for assessing energy requirement and expenditure need to be developed for use in patients with COPD. These methods need to be able to be used in the clinical setting, since the main conclusion is that calculation or prediction of energy requirements in patients with COPD with current methods has limited value.

\section{Articles in the thesis}

1. Slinde F, Grönberg AM, Engström CP, RossanderHulthén L, Larsson S. Body composition by bioelectrical impedance predicts mortality in chronic obstructive pulmonary disease patients. Respir Med (in press).

2. Slinde F, Rossander-Hulthén L. Bioelectrical impedance: effect of 3 identical meals on diurnal impedance variation and calculation of body composition. Am J Clin Nutr 2001; 74: 474-8.

3. Slinde F, Bark A, Jansson J, Rossander-Hulthén L. Bioelectrical impedance variation in healthy subjects during $12 \mathrm{~h}$ in the supine position. Clin Nutr 2003; 22: 153-7.

4. Slinde F, Ellegård L, Grönberg AM, Larsson S, Rossander-Hulthén L. Total energy expenditure in underweight patients with severe chronic obstructive pulmonary disease living at home. Clin Nutr 2003; 22: 159165.

5. Slinde F, Kvarnhult K, Grönberg AM, Nordenson A, Larsson S, Rossander-Hulthén L. Energy expenditure in underweight chronic obstructive pulmonary disease patients before and during a physiotherapy program (submitted).

\section{Frode Slinde}

E-mail: frode.slinde@nutrition.gu.se 